\title{
CPM-Based Spread Spectrum Systems for Multi-user Communications
}

\author{
Nicolò Mazzali ${ }^{a}$, Giulio Colavolpe ${ }^{a}$, and Stefano Buzzi ${ }^{b}$ \\ ${ }^{a}$ Università degli Studi di Parma, Dipartimento di Ingegneria dell'Informazione, Parma, Italy \\ ${ }^{b}$ University of Cassino, DAEIMI, Cassino, Italy
}

\begin{abstract}
We propose a new spread spectrum (SS) system based on continuous phase modulations (CPMs). The main idea is to exploit the sequence of modulation indices of a multi- $h$ CPM as a frequency hopping (FH) sequence. We will show how the sequence of indices impacts on the CPM signal bandwidth and power spectral density (PSD). Spectral spreading, flatness and smoothness can be easily achieved by an appropriate choice of the maximum value of the modulation index and of the length of the index sequence. We will show that a CPM-based spread spectrum system achieves an overall spectral efficiency larger than that of a single-user single- $h$ CPM signal even when a single-user detector is employed at the receiver.
\end{abstract}

\section{INTRODUCTION}

Modern satellite communications require modulation formats robust to nonlinearities and multiple access interference (MAI), as well as power- and spectrally efficient. Robustness to nonlinearity is mandatory in order to use strongly saturated amplifiers, and spectral efficiency is one of the most important quality figures in any communication system. For this reason the choice of using modulation formats such as continuous phase modulations (CPMs) comes quite naturally. CPMs robustness to nonlinearity stemming from the constant envelope is one of the main reasons of their popularity, along with excellent power and spectral efficiencies [1].

Code division multiple access (CDMA) is one of the most studied methods for multi-user communication systems. Based on the employed spread-spectrum (SS) technique, CDMA schemes are grouped in two major classes, namely directsequence SS (DS-SS) and frequency-hopping SS (FH-SS).

DS-SS has been combined with CPMs in many different ways. Lane and Bush [2] proposed a SS multi- $h$ (SSMH) CPM whose drawbacks in a multi-user scenario will be discussed in Section IV. Giannetti et al. [3] studied only a very special and limited subset of CPMs, known as generalized minimum shift keying (GenMSK), which can be approximately viewed as a linear modulation. Hence, classical results of DS-SS linear modulations apply. Hsu and Lehnert [4] considered a multi-user system where each user transmits a SS signal that is the product between a linear modulation (for the data) and a multi- $h$ CPM (for the spreading chips), giving up to phase continuity. This problem has been solved by Yang et al. [5] mapping the $M$-ary data symbols into $M$ phase spreading sequences (PSSs) modulated by a single- $h$ CPM modulator. The main problem of this approach is the timeconsuming design of a unique set of $M$ different and orthogonal PSSs for each user. Moreover, a simple receiver structure is not available because data and chips are not separable. The separation between data and chips has been preserved in the dual-phase (DP) technique proposed by McDowell et al. [6]. Chips are modulated as a multi- $h$ CPM, data are modulated as a MSK signal, and finally multiplied. The receiver, as in the linearly-modulated DS-SS systems, is composed by an analogical (and therefore expensive) despreader and a detector.

To our knowledge, FH has never been studied as a multiple access technique in CPM-based systems. Nevertheless, FH has been used with the purpose of spreading the CPM power spectral density (PSD) for security issues in [7]) and [8]).

Here, a new multiple access technique based on multi- $h$ CPMs is proposed. The main idea is to exploit the fact that each CPM can be viewed as a frequency modulation where the frequency deviation is strictly related to the modulation index. Since in multi- $h$ CPMs the modulation index is cyclically varied every symbol period, the resulting effect is a sort of frequency hopping. This is exactly an instance of $\mathrm{FH}$ when applied to a continuous phase FSK (CPFSK). So, we will use multi- $h$ CPM not to improve BER performance (as in [2], [4] and [6]) but to spread the PSD and allow multiple access without resorting to spreading codes. In other words, we directly construct a modulation format with a PSD extremely flat, large, and smooth at will. The correspondig single-user detector has the same complexity of the classical single- $h$ CPM detector with the same number of phase states (which is a clear advantage if compared to the complexity of the receiver structure of [5] and [6]). In CPM literature, the modulation index is hardly ever chosen bigger than one, even though this would not invalidate the CPM definition. Therefore, the most natural way to spread the CPM PSD is by using indices varying in a wide range, exploiting indices much bigger than one. Moreover, using a long sequence of indices, the CPM PSD will become smoother. Assigning to each user a different and randomly generated index sequence, we will obtain a new and efficient FH-SS technique for CPM-based systems, where the spectral spreading effect is now embedded in the modulation format itself.

\section{SYSTEM MODEL}

\section{A. CPM signal}

The complex envelope of a generic multi- $h$ CPM signal is

$$
s(t)=\sqrt{\frac{2 E_{s}}{T}} \exp \left\{\jmath\left[2 \pi \sum_{i} h_{\underline{i}} \alpha_{i} q(t-i T)+\theta\right]\right\}
$$

where $E_{s}$ is the mean energy per symbol, $T$ is the symbol period, $\left\{\alpha_{i}\right\}$ are the $M$-ary information symbols, $\left\{h_{i}\right\}$ is the 
sequence of $N_{h}$ modulation indices, $\underline{i}=i \bmod N_{h}, q(t)$ is the phase-smoothing response characterizing the format, and $\theta$ is the initial phase offset. The phase-smoothing response is a continuous function satisfying the following property:

$$
q(t)= \begin{cases}0 & \text { when } t \leq 0 \\ \frac{1}{2} & \text { when } t \geq L T\end{cases}
$$

$L$ being the correlation length of the signal. The frequency pulse is defined as

$$
p(t)=\frac{\mathrm{d}}{\mathrm{d} t} q(t)
$$

and (1) can be rewritten as

$$
s(t)=\sqrt{\frac{2 E_{s}}{T}} \exp \left\{\jmath\left[2 \pi \int_{-\infty}^{t} \sum_{i} h_{\underline{i}} \alpha_{i} p(\tau-i T) \mathrm{d} \tau+\theta\right]\right\}
$$

which is the expression of a frequency-modulated signal using a pulse amplitude modulation (PAM) with shaping pulse $p(t)$ as modulating signal. The most used frequency pulses are the rectangular pulse ( $L$-REC to denote its duration of $L$ symbol periods) and the raised-cosine pulse ( $L-\mathrm{RC})$.

CPMs are modulations with memory. In the generic symbol interval $n T \leq t<(n+1) T$, the CPM signal (1) is completely defined by symbol $\alpha_{n}$ and state $\sigma_{n}=\left(\omega_{n}, \varphi_{n}\right)$, where $\omega_{n}=$ $\left(\alpha_{n-L+1}, \ldots, \alpha_{n-1}\right)$ is the correlative state and

$$
\begin{aligned}
\varphi_{n} & =\left(\pi \sum_{i=-\infty}^{n-L} h_{\underline{i}} \alpha_{i}\right) \bmod 2 \pi \\
& =\left(\varphi_{n-1}+\pi h_{\underline{n-L}} \alpha_{n-L}\right) \bmod 2 \pi
\end{aligned}
$$

is the phase state. The correlative state can assume $M^{L-1}$ values, whereas the phase state can assume $p$ values, having defined $h_{i}=k_{i} / p$ where $k_{i}$ and $p$ are positive integer numbers ${ }^{1}$ and integer values for $h_{i}$ are forbidden. Therefore, the total number of states is $p M^{L-1}$.

\section{B. $S S-F H-C P M$}

In the proposed multi-user system, multiple access is guaranteed by assigning a different sequence of modulation indices to each user. We assume that each user transmits $K$ symbols, and we denote by $\alpha_{n}^{(u)}$ and $\sigma_{n}^{(u)}$ the symbol transmitted by user $u$ at discrete-time $n$ and the corresponding state. We define $\boldsymbol{\alpha}^{(u)}=\left(\alpha_{0}^{(u)}, \ldots, \alpha_{K-1}^{(u)}\right)^{T}$ as the vector of the $K$ symbols transmitted by user $u$, and also $\boldsymbol{\alpha}_{n}=\left(\alpha_{n}^{(1)}, \ldots, \alpha_{n}^{(U)}\right)^{T}$ as the vector of all symbols transmitted at discrete-time $n$ (one symbol per user), and $\boldsymbol{\alpha}=\left(\boldsymbol{\alpha}_{0}^{T}, \ldots, \boldsymbol{\alpha}_{K-1}^{T}\right)^{T}$, where $(.)^{T}$ denotes transpose. Similarly, we define $\boldsymbol{\sigma}_{n}=\left(\sigma_{n}^{(1)}, \ldots, \sigma_{n}^{(U)}\right)^{T}$

\footnotetext{
${ }^{1} \mathrm{~A}$ correct definition of the modulation index requires that $k_{i}$ and $p$ are relative prime to have a minimal trellis representation. As it will be clear later, the considered sequence of indices is chosen such that $p$ is kept constant whereas $k_{i}$ is chosen randomly with the only constraint that $h_{i}$ cannot be integer. When $k_{i}$ and $p$ are not relative prime, we still use, for simplicity, a trellis representation with $p$ states although it could be reduced. This allows to always use the same trellis without the need to resort to a time-varying trellis.
}

and $\boldsymbol{\sigma}=\left(\boldsymbol{\sigma}_{0}^{T}, \ldots, \boldsymbol{\sigma}_{K-1}^{T}\right)^{T}$. We also define

$$
\begin{aligned}
& s^{(u)}\left(\boldsymbol{\alpha}^{(u)}, t\right)= \\
& \sqrt{\frac{2 E_{s}^{(u)}}{T}} \exp \left\{\jmath\left[2 \pi \sum_{i=0}^{K-1} h_{\underline{i}}^{(u)} \alpha_{i}^{(u)} q(t-i T)+\theta^{(u)}\right]\right\}
\end{aligned}
$$

the signal transmitted by user $u$ and, without loss of generality, we assume that all users employ the same values of $T, M, L$, $p, N_{h}$ and $h_{\max }, h_{\max }$ being the maximum value taken by the modulation index. We will also assume that all users employ the same phase-smoothing response $q(t)$.

We consider an asynchronous multiple-access system on an additive white Gaussian noise (AWGN) channel, so that the complex envelope of the received signal is

$$
\begin{aligned}
r(t) & =\sum_{u=1}^{U} s^{(u)}\left(\boldsymbol{\alpha}^{(u)}, t-\tau^{(u)}\right)+w(t) \\
& =s^{(\ell)}\left(\boldsymbol{\alpha}^{(\ell)}, t-\tau^{(\ell)}\right)+\sum_{\substack{u=1 \\
u \neq \ell}}^{U} s^{(u)}\left(\boldsymbol{\alpha}^{(u)}, t-\tau^{(u)}\right)+w(t)
\end{aligned}
$$

Initial phase offsets $\theta^{(u)}$ and delays $\tau^{(u)}$ are random variables uniformly distributed in $[0,2 \pi)$ and $[0, T)$, respectively. The thermal noise is a zero-mean circularly symmetric white Gaussian process with PSD $2 N_{0}$.

Fixing the indices denominator $p$ is mandatory to keep constant the number of the phase states, while fixing the maximum numerator allows every user to undergo the same spectral spreading. Each user has a different sequence of randomlygenerated modulation indices. The spectral spreading depends only on the range of values assumed by the modulation index, the larger this range, the stronger the spreading effect. The number of modulation indices $N_{h}$ plays a role only in the smoothness of the PSD. A CPM with high $N_{h}$ will show a smooth PSD with small oscillations and no sidelobes.

The number of users allowed in the system depends on the total number of possible indices $\nu=p h_{\max }-\left\lfloor h_{\max }\right\rfloor$ (where $\lfloor x\rfloor$ denotes the maximum integer lower than $x$ ). If we impose the absence of overlaps, in a synchronous system the maximum number of users would coincide with the number of possible indices

$$
U_{\max }=\nu .
$$

In practical receivers, an approximated set of sufficient statistics for MAP symbol detection is obtained as described in [9]. We assume the useful signal component to be bandlimited-although this is an approximation in the case of CPM signals, whose PSD has an infinite support-with bandwidth lower than $N / 2 T$, where $N$ is a proper positive integer. The approximated statistics can be obtained by extracting $N$ samples per symbol interval from the received signal (5) prefiltered by means of a low-pass filter which leaves unmodified the useful signal and has a vestigial symmetry around $N / 2 T$. The condition on the vestigial symmetry ensures that the noise samples are independent and identically distributed 
complex Gaussian random variables with independent components. We denote with $r_{n, m}$ the $m$-th received sample (with $m=0, \ldots, N-1$ ) of the $n$-th symbol interval, with $\mathbf{r}_{n}=\left(r_{n, 0}, \ldots, r_{n, N-1}\right)^{T}$ the vector of the received samples in the $n$-th symbol interval, and with $\mathbf{r}=\left(\mathbf{r}_{0}^{T}, \ldots, \mathbf{r}_{K-1}^{T}\right)^{T}$ the vector of all received samples,

When considering coded CPM schemes where the CPM modulator is concatenated, possibly through an interleaver, with an outer encoder (as an example, see [10] and references therein), the receiver is usually based on a soft-input soft-output (SISO) detector that iteratively exchanges softinformation with the outer SISO decoder according to the turbo principle. Regarding single-user SISO CPM detection, little can be added to what already said in the literature (as an example, see [11] and references therein) - the adoption of multi- $h$ CPM signals here entails only trivial modifications with respect to the case of single- $h$ CPMs or the adoption, in case of simplified detectors, of the Laurent decomposition extended to multi- $h$ signals [12]. Suboptimal multi-user SISO CPM detectors ${ }^{2}$ can also be conceived, by extending those described in [13] for frequency-division-multiplexed CPM systems. The derivation of the algorithms is not shown here for a lack of space.

\section{SPECTRAL EFFICIENCY}

The main quality figure we consider in this work is the overall spectral efficiency $\eta_{U}$ of the system. Since we are considering a CDMA scenario, the most intuitive way to compute $\eta_{U}$ is to evaluate the spectral efficiency $\eta$ of a reference user, and then define $\eta_{U}=U \eta$.

The spectral efficiency for the reference user can be computed as

$$
\eta=\frac{I}{B T} \quad[\mathrm{bit} / \mathrm{s} / \mathrm{Hz}]
$$

where $B$ is the bandwidth occupied by the CPM signal and $I$ is the information rate of the user. CPM bandwidth is theoretically infinite because the PSD of a CPM signal has rigorously an infinite support. Hence, we adopted the traditional definition of bandwidth based on the power concentration, that is the bandwidth that contains a given fraction of the overall power. Being this fraction a parameter, we chose to use the $99.99 \%$ of the overall power. CPM power spectral density cannot be evaluated analytically in closed form but only numerically. The adopted algorithm is the one proposed in [14], [15].

To compute the information rate $I$ for the reference user, we can use the simulation-based technique described in [16], which only requires the existence of an optimal MAP symbol detector for the considered system. Unfortunately, the complexity of the optimal MUD is exponential in $U$, making the evaluation of $I$ practically unfeasible. Therefore, we can evaluate an achievable lower bound by resorting to the concept of mismatched detection [17]. We can consider an approximated channel model (the auxiliary channel) for which an exact MAP symbol detection with affordable complexity exists - the more

\footnotetext{
${ }^{2}$ The optimal multi-user detector (MUD), although known in principle, has an exponential complexity in the number of users $U$ and is thus unfeasible.
}

similar the auxiliary channel to the actual channel, the tighter the obtained bound on the spectral efficiency.

As done in [1], we approximate the channel model at the receiver side by modelling the interference as a zero-mean circularly symmetric white Gaussian process with PSD $2 N_{I}$, $N_{I}$ being a design parameter independent of the thermal noise and optimized via numerical simulations. This approximation is exploited only by the receiver, while in the actual channel the interference is generated as in (5). Hence, the considered auxiliary channel model is that for which the received signal reads

$$
r(t)=s^{(\ell)}(t)+\zeta(t)
$$

where $\zeta(t)$ is a zero-mean circularly symmetric white Gaussian process with PSD $2\left(N_{0}+N_{I}\right)$. The simulation-based method described in [16] allows to evaluate the achievable information rate for the mismatched receiver, i. e.,

$$
I\left(\boldsymbol{\alpha}^{(\ell)}, \mathbf{r}\right)=\lim _{J \rightarrow \infty} \frac{1}{J} \mathrm{E}\left\{\log \frac{p\left(\mathbf{r}^{J} \mid \boldsymbol{\alpha}^{(\ell) J}\right)}{p\left(\mathbf{r}^{J}\right)}\right\} \quad[\text { bit/ch.use }]
$$

where we used the superscript $J$ to remark that a sequence is truncated to its first $J$ elements. In (8) $p\left(\mathbf{r}^{J} \mid \boldsymbol{\alpha}^{(\ell) J}\right)$ and $p\left(\mathbf{r}^{J}\right)$ are PDFs according to the auxiliary channel model (7), while the statistical average is with respect to the input and the output sequences evaluated according to the actual channel model (5). Both $p\left(\mathbf{r}^{J} \mid \boldsymbol{\alpha}^{(\ell) J}\right)$ and $p\left(\mathbf{r}^{J}\right)$ can be evaluated recursively through the forward recursion of the MAP detection algorithm matched to the auxiliary channel model [16]. The mismatched receiver can assure error-free transmissions when the transmission rate at the CPM modulator input does not exceed $I\left(\boldsymbol{\alpha}^{(\ell)}, \mathbf{r}\right)$ bits per channel use.

\section{NUMERICAL RESULTS}

We consider an asynchronous SS-FH-CPM system using binary data symbols $(M=2)$, a $2-\mathrm{RC}$ frequency pulse, $N_{h}=16, p=8$ and $h_{\max }=39 / 8$. Since we are not interested in a particular sequence of indices but in the average behaviour of the system, we consider a packet transmission (with 1024 symbols per packet) and, for each user, we change the sequences of indices $\left\{h_{i}^{(u)}\right\}_{i=0}^{N_{h}-1}$, the time delays $\tau^{(u)}$ and the initial phase offsets $\theta^{(u)}$ every packet. Since the sequence of indices is randomly generated, the signal bandwidth $B$ is a random variable too. Hence, we evaluated the average PSD over a thousand different sequences of indices in order to find the mean and the variance of $B$.

As shown in Fig. 1, the average PSD is extremely smooth and the normalized bandwidth has mean $B T=6.66$ and variance $\sigma_{B T}^{2}=0.096$. In Fig. 1, we also show the PSD of the unspread signal used in a SSMH system [2]. The frequency pulse is $2-\mathrm{RC}$ and two modulation indices, $1 / 2$ and $5 / 8$, have been adopted.

Let us now compare the spectral efficiency of different systems. We first compare the proposed SS-FH-CPM system with a couple of SSMH systems in [2], then with different single- $h$ systems. In a multi-user scenario, the SSMH-CPM systems in [2] need the use of spreading sequences of length $N_{c}$ chips per symbol period, with $N_{c} \geq U$ (even though 


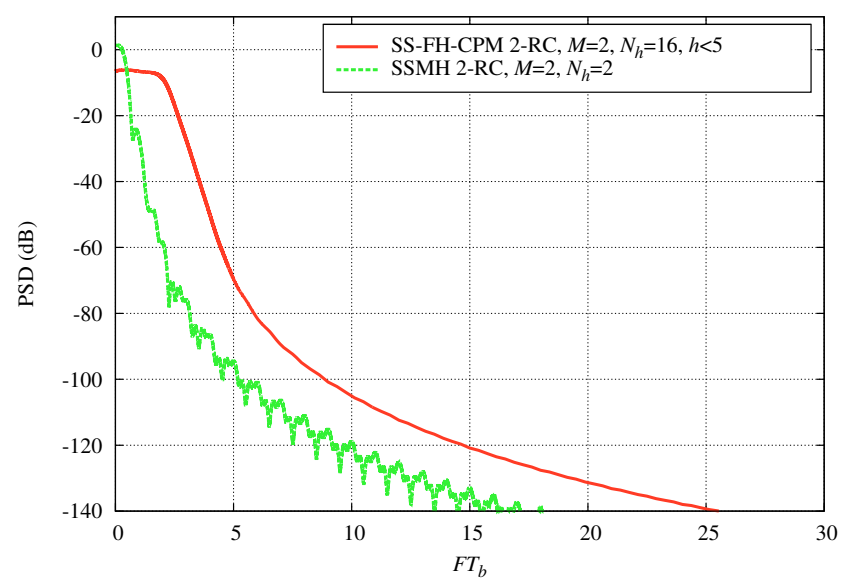

Figure 1. Power spectral density of the considered 2-RC binary SS-FH-CPM with $N_{h}=16, p=8, h_{\max }=39 / 8$, and of the binary 2-RC SSMH scheme with $\left\{h_{i}\right\}=\{1 / 2,5 / 8\}$ in [2].

Table I

BANDWIDTHS OF THE CONSIDERED 2-RC BINARY SS-FH-CPM WITH $N_{h}=16, p=8, h_{\mathrm{MAX}}=39 / 8$, AND OF THE BINARY 2 -RC SSMH SCHEME WITH $\left\{h_{i}\right\}=\{1 / 2,5 / 8\}$ IN [2].

\begin{tabular}{|c|c|}
\hline modulation format & $B T$ \\
\hline \hline SS-FH 2-RC & 6.66 \\
\hline SSMH 2-RC $\left(N_{c}=1\right)$ & 2.07 \\
\hline SSMH 2-RC $\left(N_{c}=3\right)$ & 6.22 \\
\hline SSMH 2-RC $\left(N_{c}=4\right)$ & 8.3 \\
\hline
\end{tabular}

some overload is possible). The normalized bandwidth of the unspread signal is then multiplied by a factor $N_{c}$, and therefore the global spectral efficiency is very low. As a benchmark, we consider the SSMH-CPM system already considered in Fig. 1. For this SSMH-CPM system, the used spreading sequences are random binary sequences generated every packet period together with the time delays and the initial phase offsets. We will show the maximum spectral efficiencies $\eta_{U}$ achievable by the SSMH system (obtained by the joint optimization of $U$ and $N_{I}$ ) with $N_{c}=3$ and $N_{c}=4$. We chose these two values of spreading factor to compare our SS-FH-CPM system to two SSMH systems with similar bandwidth, as shown in Table I. Figure 2 shows the spectral efficiency $\eta_{U}$ as a function of the ratio $E_{b} / N_{0}, E_{b}$ being the received signal energy per information bit. It is easy to see that the SSMH system has values of $\eta_{U}$ much lower than that of the proposed SS-FH system, for which the considered number of users $U$ has been found jointly maximizing $\eta_{U}$ as a function of the number of users $U$ and the interference noise variance $N_{I}$ for a fixed signal-to-noise ratio (SNR) value. We also considered $N_{c}=16$ (in analogy to the length of the indices sequences of the SS-FH system $N_{h}$ ), but the maximum achievable $\eta_{U}$ fell down to zero. The same happened with $U=30$ users. These considerations prove that the SSMH technique is not suitable for multi-user systems.

In order to make some other comparisons with the proposed SS-FH-CPM system, we also considered single-user systems using binary single- $h$ CPMs with a 2 -RC frequency pulse and
Table II

BANDWIDTHS OF SINGLE- $h$ 2-RC CPMS WITH DIFFERENT MODULATION INDICES

\begin{tabular}{|c|c|}
\hline$h$ & $B T(2-\mathrm{RC})$ \\
\hline \hline $1 / 8$ & 1.33 \\
\hline $4 / 8$ & 2.15 \\
\hline $7 / 8$ & 2.95 \\
\hline
\end{tabular}

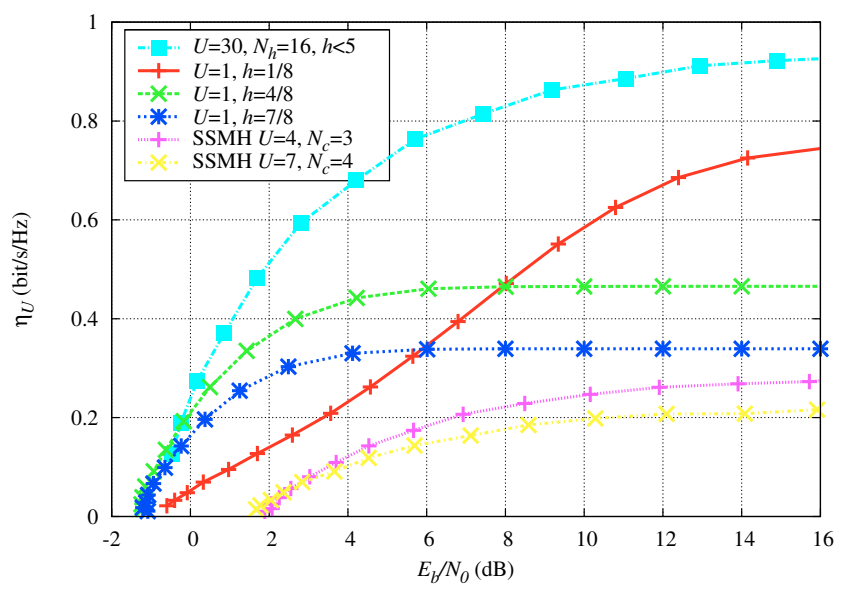

Figure 2. Spectral efficiencies of the considered 2-RC binary SS-FH-CPM with $N_{h}=16, p=8, h_{\max }=39 / 8$, of different single- $h$ 2-RC CPMs with $h=1 / 8, h=4 / 8$, and $h=7 / 8$, respectively, and of the binary 2 -RC SSMH scheme with $\left\{h_{i}\right\}=\{1 / 2,5 / 8\}$ in [2] with $N_{c}=3$ and $U=4$, and with $N_{c}=4$ and $U=7$.

$h<1$, as traditionally done in literature. There is no interest in considering single- $h$ systems with $h>1$ because they have a larger bandwidth than those with $h<1$, resulting in a lower spectral efficiency. For the single- $h$ systems, the signal bandwidth strongly depends on $h$ (as shown in Table II), and so does the spectral efficiency. Hence, we chose to show the highest (at high signal-to-noise ratio values), an intermediate, and the lowest curves of spectral efficiency, obtained with $h=1 / 8, h=4 / 8$, and $h=7 / 8$ respectively. As it can be seen in Fig. 2, the SS-FH-CPM system has a spectral efficiency that surpasses that of all single-user single- $h$ systems.

For the proposed system, the curve in figure 2 proves that an error-free communication, at the obtained spectral-efficiency values, is possible, even with $U \gg 1$, using a single-user detector (SUD) and a proper channel coded scheme. BER performance curves, not shown here for a lack of space, confirm these results. In order to assess the performance of the suboptimal MUDs previously mentioned, we consider a coded SS-FH-CPM system with $U=3$ synchronous users using binary 2-RC CPM with $p=4, h_{\max }=19 / 4, N_{h}=8$. Each user has the same power (i.e., $E_{s}^{(1)}=E_{s}^{(2)}=\ldots=$ $\left.E_{s}^{(U)}=E_{s}\right)$ and uses the $(64,51) \mathrm{eBCH}$ code with rate $R=0.79$ and random interleaver described in [10]. As for the spectral efficiency, since we are not interested in a particular index sequence but in the average behaviour of the system, we considered a packet transmission (with 128 symbols per packet) and changed the index sequences $\left\{h_{i}^{(u)}\right\}_{i=0}^{N_{h}-1}$, the initial phase offsets $\theta^{(u)}$, and the code interleaver every packet 
for each user. As a benchmark, we consider the bit error rate (BER) of a SUD with $U=3$ users and the BER of a SUD in absence of interference ( $U=1$ user). For each detector, we increased the noise variance used by the receiver to $2\left(N_{0}+N_{I}\right) N / T$, where $N_{I}$ is an optimized parameter aiming to reduce the overestimated confidence of the BCJR algorithm [13]. Being the MUDs iterative, we considered 20 iterations between detector and decoder.

For the described suboptimal multiuser detectors, the performance also depends on the adopted schedule. Serial or parallel schedules are usually considered in the literature. For a lack of space, and also because the difference in performance is practically negligible in this scenario of users transmitting at the same power, we only consider the parallel schedule. In this case, at each iteration all users are activated simultaneously. The computed soft-outputs are then provided to the other users for the next iteration and, after deinterleaving, to the decoders.

In the figure, we considered the detectors that in [13] are called SIC 1 and SIC 2 (see [13] for the relevant description), and that based on factor graphs and the sum-product algorithm (FG in the figure) originally proposed in [13]. Since SIC 1 and SIC 2 detectors show the same performance when the users are uncorrelated (or weakly correlated), we decided to introduce a correlation to point out the different behaviour of the two algorithms. Therefore we generate the index sequence for user $u=1$ randomly, and from that we derive all the other sequences as

$$
h_{i}^{(u)}=h_{i}^{(1)}+\frac{u-1}{p} .
$$

If $h_{i}^{(u)}$ is an integer then we change its value in $h_{i}^{(1)}+u / p$. The relevant performance is shown in figure 3. Clearly, since the users are strongly correlated, the SUD fails. The SIC 2 algorithm performs much better but, as expected, even better does the SIC 1. However, the FG receiver exhibits the best performance since the Gaussian approximation of the interference, over which SIC 1 and SIC 2 are based, is not accurate with only two interferers. To see the SIC algorithms outperform the FG receiver, we should have considered a higher number of users, that unfortunately would have made the simulations extremely burdensome and slow.

\section{CONCLUSions}

We proposed a brand new technique allowing to use multi$h$ CPM in CDMA systems. Tuning the highest value the modulation index can assume, it is possible to set the spectral spreading of the CPM signal. PSD smoothness is reachable using a long enough sequence of modulation indices. In a multi-user scenario, the proposed system can surpass the spectral efficiency of a single-user single- $h$ system, whereas BER performance can be improved by a suboptimal MUD.

\section{REFERENCES}

[1] A. Barbieri, D. Fertonani, and G. Colavolpe, "Spectrally-efficient continuous phase modulations," IEEE Trans. Wireless Commun., vol. 8, pp. 1564-1572, Mar. 2009.

[2] W. Lane and A. Bush, "Spread-spectrum multi-h modulation," Selected Areas in Communications, IEEE Journal on, vol. 8, no. 5, pp. $728-742$, jun 1990.

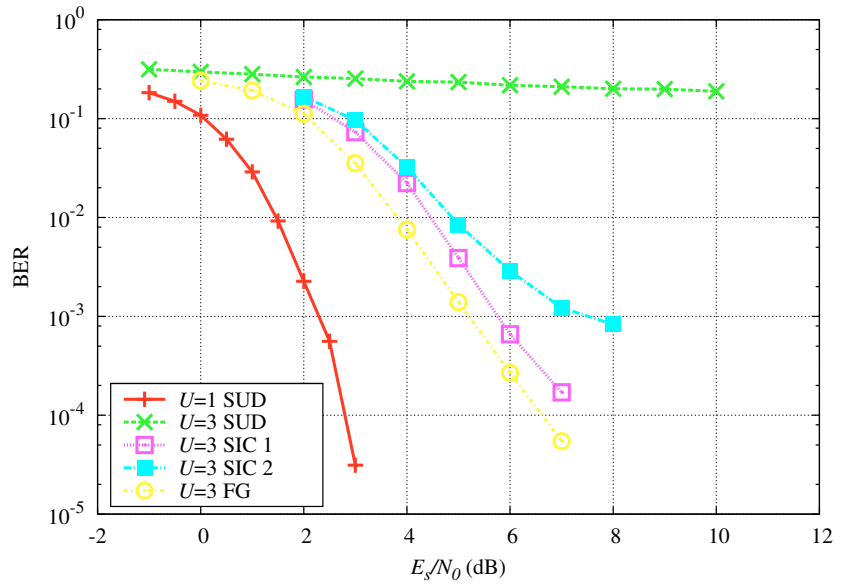

Figure 3. BER of binary 2-RC systems with $U=1, U=3$ with a SUD and the different MUDs, with $N_{h}=8, p=4, h_{\max }=19 / 4$, and a $(64,51)$ eBCH code with rate $R=0.79$.

[3] F. Giannetti, M. Luise, and R. Reggiannini, "Continuous-phase modulations for CDMA radio communications: Modem architecture and performance," European Transactions on Telecommunications, vol. 7, no. 3, pp. 225-233, 1996. [Online]. Available: http://dx.doi.org/10.1002/ett.4460070302

[4] R. Hsu and J. Lehnert, "The performance of continuous-phase-coded DS/SSMA communications," Communications, IEEE Transactions on, vol. 46, no. 4, pp. $533-543$, apr 1998.

[5] F. Yang, S. Leung, C. Ngan, and G. Bi, "The performance and design criterion of phase spreading sequences for DS/SSMA communications with full response CPM over Rayleigh fading channels," in Global Telecommunications Conference, 1999. GLOBECOM '99, vol. 1B, 1999, pp. $914-918$ vol. $1 \mathrm{~b}$.

[6] A. McDowell, J. Lehnert, and Y. Jeong, "Dual-phase continuous phase modulation for spread-spectrum multiple-access communication," Communications, IEEE Transactions on, vol. 52, no. 5, pp. 823 - 833, may 2004.

[7] P. Voglewede, "Frequency hopping with multih CPM (MIL-STD-188181B)," in Military Communications Conference, 2003. MILCOM 2003. IEEE, vol. 2, oct. 2003, pp. 1089 - 1094 Vol.2.

[8] C. Brown and P. Vigneron, "Spectrally efficient CPM waveforms for narrowband tactical communications in frequency hopped networks," in Military Communications Conference, 2006. MILCOM 2006. IEEE, oct. 2006, pp. $1-6$.

[9] H. Meyr, M. Oerder, and A. Polydoros, "On sampling rate, analog prefiltering, and sufficient statistics for digital receivers," IEEE Trans. Commun., vol. 42, pp. 3208-3214, Dec. 1994.

[10] A. Graell i Amat, C. A. Nour, and C. Douillard, "Serially concatenated continuous phase modulation for satellite communications," IEEE Trans. Wireless Commun., vol. 8, pp. 3260-3269, Jun. 2009.

[11] A. Barbieri and G. Colavolpe, "Simplified soft-output detection of CPM signals over coherent and phase noise channels," IEEE Trans. Wireless Commun., vol. 6, no. 7, pp. 2486-2496, Jul. 2007.

[12] E. Perrins and M. Rice, "PAM decomposition of $M$-ary multi- $h$ CPM," IEEE Trans. Commun., vol. 53, no. 12, pp. 2065-2075, Dec. 2005.

[13] A. Piemontese and G. Colavolpe, "A novel graph-based suboptimal multiuser detector for FDM-CPM transmissions," IEEE Trans. Wireless Commun., vol. 9, pp. 2812-2819, Sep. 2010.

[14] M. Luise, "Easy calculation of power spectra for multi-h phase-coded signals," Electronics Letters, vol. 21, no. 14, pp. 608 -609, 41985.

[15] _ "Erratum: Easy calculation of power spectra for multi-h phasecoded signals," Electronics Letters, vol. 21, no. 15, p. 661, 181985.

[16] D. M. Arnold, H.-A. Loeliger, P. O. Vontobel, A. Kavčić, and W. Zeng, "Simulation-based computation of information rates for channels with memory," IEEE Trans. Inform. Theory, vol. 52, no. 8, pp. 3498-3508, Aug. 2006.

[17] N. Merhav, G. Kaplan, A. Lapidoth, and S. Shamai, "On information rates for mismatched decoders," IEEE Trans. Inform. Theory, vol. 40, no. 6, pp. 1953-1967, Nov. 1994. 